\title{
Table Structure Recognition Based on Cell Relationship, a Bottom-Up Approach
}

\author{
Darshan Adiga Shabir Bhat Muzaffar Shah Viveka Vyeth \\ Datoin, Bengaluru, India \\ firstname. lastnameddatoin.com
}

\begin{abstract}
In this paper, we present a relationship extraction based methodology for table structure recognition in PDF documents. The proposed deep learning-based method takes a bottom-up approach to table recognition in PDF documents. We outline the shortcomings of conventional approaches based on heuristics and machine learningbased top-down approaches. In this work, we explain how the task of table structure recognition can be modeled as a cell relationship extraction task and the importance of the bottom-up approach in recognizing the table cells. We use Multilayer Feedforward Neural Network for table structure recognition and compare the results of three feature sets. To gauge the performance of the proposed method, we prepared a training dataset using 250 tables in PDF documents, carefully selecting the table structures that are most commonly found in the documents. Our model achieves an overall accuracy of $97.95 \%$ and an F1-Score of $92.62 \%$ on the test dataset.
\end{abstract}

\section{Introduction}

Usage of digital documents have elevated drastically over the last two decades and a need for automatic information extraction from these documents has increased. Portable Document Format (PDF) has been introduced by Adobe in 1993. PDF documents are the most common format of digital documents and are extensively used in scientific research, finance, enterprises etc. As the production and usage of PDF documents have increased massively, substantial research work has focused on automating the methods for docu- ment analysis (Correa and Zander, 2017; Kavasidis et al., 2018).

Tabular data is a powerful way to represent the data, among other elements of a document like charts, images etc. Tables are found in a variety of classes of digital documents and are very useful to readers to capture, search and compare the facts, summarizations and draw conclusions. Automatically extracting the information from the tables and representing the information in more convenient formats for digital consumption add immense value in the field of document understanding (Gilani et al., 2017; Hao et al., 2016).

Tables contain structured data but often are rendered as semi-structured and unstructured on the digital documents for human consumption. Data can be represented using a variety of layouts in tables without losing the meaning of data (Anand et al., 2019). The layout of tables can vary in alignment, line and word spaces, column and row spans, borders and other styling information. Depending on the type of documents and authors, the tables may not contain any border lines and the structure of the tables will still be understandable to readers. The data represented by the tables in itself can have different semantics. For example, in a table, a column may contain a list of prices in dollars, indicating that all the values of that column contain numeric data only. Similar semantic information is embedded in the table rows as well. Further, a column may have multiple subcolumns, making the original column to span multiple table cells horizontally. In rare cases, rows can also span multiple table cells vertically. All these characteristics of a table make the automatic extraction of table information more challenging.

Table extraction is a sub-problem of document understanding, that deals with information extraction and representation of tabular data. Extraction of information from tables in documents has chal- 
lenged the researchers over the last two decades. An ample amount of research work has been carried out leading to a diverse list of approaches including heuristics, rule-engine, and recently machine learning based proposals.

We believe, generalizing the patterns across the variety of table layouts in diverse type of documents, is best solved by machine learning approach. We propose a bottom-up approach for table structure recognition as a cell relation extraction task between the table text tokens using deep learning. The way a human understands the tables can be analogous to the proposed approach. Often, relation extraction task involves classification of an entity pair to a set of known relations, using documents containing mentions of the entity pair (Kumar, 2017). By considering the table recognition task as a relationship extraction problem, we introduce a novel approach suitable for several document understanding solutions.

The proposed method deals with the basic building blocks of any table, the table cells. With this approach, we hope to solve the column and row spanning, the presence or absence of borders, and other challenges mentioned earlier. The table recognition system operates at token-level and involves learning the complex patterns in order to extract the cell relationships among the table text tokens using deep learning.

\section{Related Work}

According to the well-known ICDAR 2013 Table Competition (Gbel et al., 2013), the problem of table understanding can be split into table location detection, table structure recognition, and table interpretation. Each of these sub-problems has attracted a great deal of attention from researchers and has extensive work.

A peek at the literature shows that many heuristic solutions have been proposed for table structure recognition. Most of those work consider the white space and layout analysis. Yildiz et al. (2005) propose an algorithm to recognize the columns of a table using distances between lines and then identify the cells to find the rows. The algorithm makes a few assumptions about the structure of the tables. The work of Krüpl and Herzog (2006) takes a bottom-up approach towards structure recognition using heuristics but works on browser-rendered documents. The methodology aggregates words into columns by considering the spatial distance of neighboring words.

Klampfl et al. (2014) experimented with two unsupervised approaches for table recognition and showcased the importance of spatial distances between words of a table using vertical and horizontal histogram projection of words coordinates.

Experiments using rule-engine has been proposed by Shigarov (2015), considers the physical layout of a rendered table, and the logical layout representing the relationships between the elements of a table, differently. Another work of Shigarov et al. (2016) shows promising results in recognizing the columns and rows of tables by using the word and line distances, the order of appearance of text chunks. The methodology makes use of configurable thresholds in its heuristic decision making.

The heuristic and rule-based solutions make various assumptions on the visual, type and content, structural details of tables and the thresholds used in the algorithms. These assumptions may not hold on heterogeneous documents and may even break the system.

Perez-Arriaga et al. (2016) have made use of both k-nearest neighbor and layout heuristics, making it a hybrid methodology to recognize the table structure. The method groups the words into rows and columns using spatial distances of words heuristically. Interestingly, the spatial distance thresholds are learned using the k-nearest neighbor algorithm. Their work also proposes a heuristic method to identify the headers of the table. Deep learning based semantic segmentation has been used by Schreiber et al. (2017) where an image of a document is fed to the neural network to identify the rows and columns of a table. However, the work makes use of a heuristic post-processing step to improve the table structure recognition.

Clinchant et al. (2018) have made an extensive comparison of three different Machine Learning approaches to recognize the table structure in hand-written register books. The method first recognizes the cell locations and then groups the cells into rows. The experimentations do a thorough comparison of CRF, a variation of Graph-CN called Edge-CN, and conventional Logistic Regression algorithms. However, the method works on already recognized headers and columns of the table and addresses only row recognition task.

To the best of our knowledge, most of the re- 
lated work of table recognition try to identify the columns and rows of tables first and then locate the intersections of rows and columns as table cells. A few heuristics based works have considered the grouping of words into blocks and then aggregating blocks into rows and columns. A common downside of these methodologies is that they fail to capture the information about rows and columns spanning multiple table cells. Few of the heuristic approaches do try to solve this issue however, they fail to generalize the solution.

We propose a purely bottom-up approach by building the table structure by recognizing the individual cells of the table and their location in the document. The task of recognizing the table cells is addressed as cell-relation extraction between the tokens present in the table.

\section{Methodology}

In this section, we first explain how we modeled the table structure recognition as a relation extraction task, then the training data preparation, and finally describe how the binary relationship classification is modeled using a Multilayer Feedforward Neural Network.

\subsection{Cell Relationship Extraction in Table Structure Recognition}

Humans will recognize the table structure even without a need for borders, based on visual clues, spatial distances and the content of the cells. These visual clues present in the tables help the readers to recognize the location of table cells easily, by bringing all the words of a cell together both visually and semantically. The proposed method is based on this idea of identification of cell relationship among the table words. The first step towards the recognition of rows or columns is the identification of table cells and thus the whole process of table structure recognition is a bottom-up process. This reasoning is based on the underlying definition of any table: Unit of a table is a cell, horizontal and vertical alignment of cells forms rows and columns, respectively. Tokens are generated by using white-space and new-line characters as delimiters. In this paper the terms token and word are used interchangeably.

Relation extraction is a well-known task in Natural Language Processing, which deals with classifying whether a given set of $n$ samples have any of $m$ different relationships. For example, in lin- guistics, determining whether two or more expressions in a text refer to the same person or thing, is a relation extraction task. We take the idea of relation extraction and formulate the task of table structure recognition as identifying the cellrelationship among all the content of individual cells of a table. When the tokens that are part of a table are considered as the smallest possible elements of a table, the relation extraction task will be to identify whether given two tokens of table text, belong to the same cell or not. If two tokens belong to the same cell, then those two tokens have a belong-to-same-cell relation. In our experiments, this task of binary relationship extraction is considered as a binary classification problem.

For every pair of tokens, the goal of binary relationship classifier is to determine whether the two tokens belong to the same cell or not. An important thing to note here is that this relationship between the two tokens is transitive. If a token $\mathrm{A}$ is related to the token $B$ and the token $B$ is related to the token $\mathrm{C}$, then the token $\mathrm{A}$ is related to $\mathrm{C}$. Hence, we don't need to generate feature vectors of all the possible pairs of tokens in a cell to determine all the tokens of a cell, we only need to make sure that all the tokens of a cell are connected via a chain of such transitive dependencies.

Once we predict the belong-to-same-cell relation between token pairs, we group all the table tokens into different cells. This is a simple task of aggregating different token pairs into their respective cells. Using this data of all the table cells and the tokens in each of the cells, we can model the recognition of rows and columns of the table again as a relation extraction task between the pairs of table cells themselves. However, in this work, we concentrate only on cell recognition.

\subsection{Data Preparation}

\section{Detecting Tables and Training Data Generation}

The detection of the location of tables in PDF documents is the first task in the process of table extraction and this location information is prerequisite to our system. There are several open source and free of charge tools for detecting table locations in PDF documents. In our experiments, we used Tabula to obtain the location details of a table in the document. The location of a table is represented by five values, pageNum, (startX, startY) and (end $X$, end $Y$ ). All the coordinates are assigned 


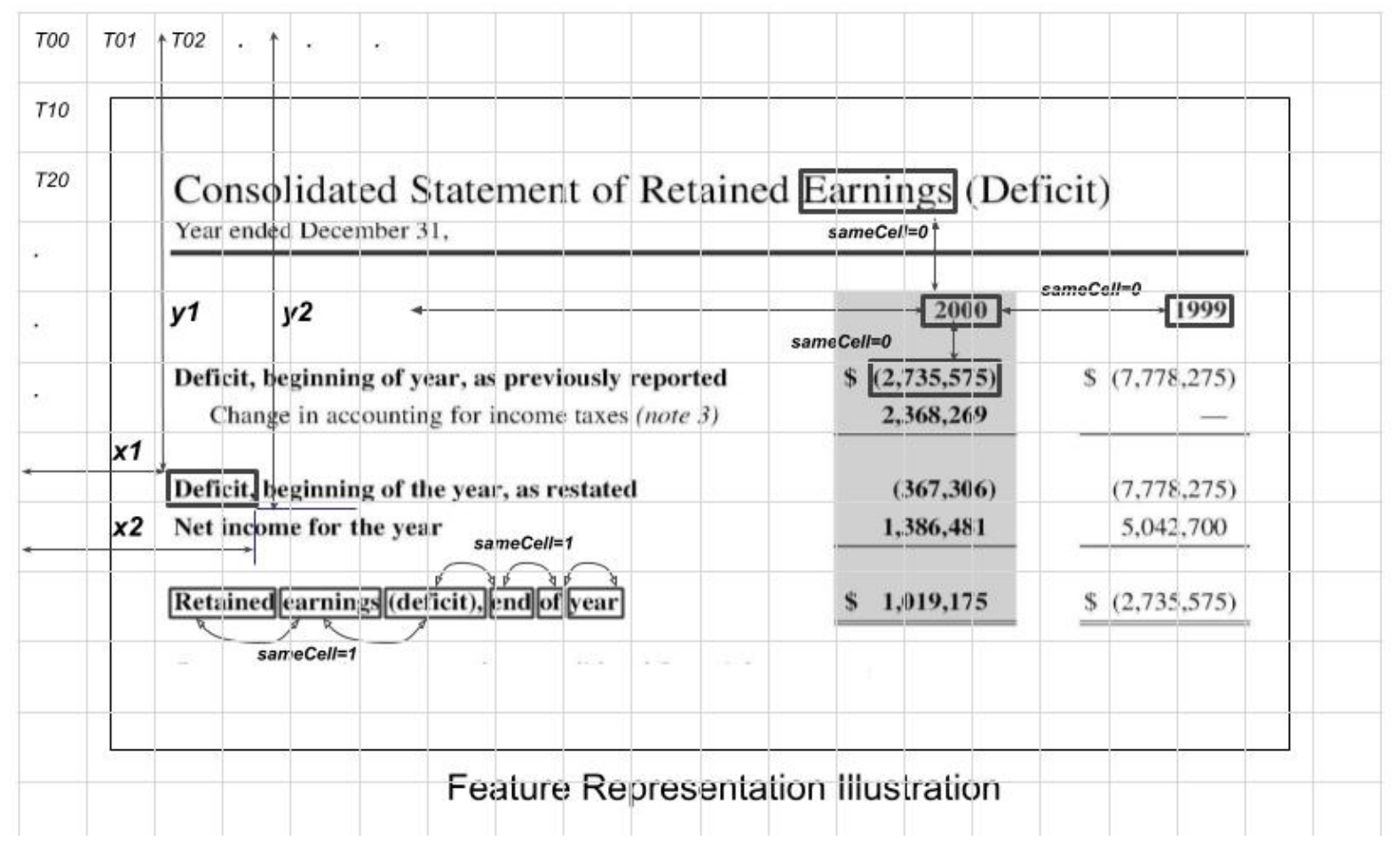

Figure 1: Features used in cell-relation extraction. A) T00, T01, T02, .. are the tiles in the first row of tile matrix. B) T00, T10, T20,... are the tiles in the first column of tile matrix. C) (x1,y1) and (x2,y2) are the start and end coordinates of a token. D) sameCell=1, Pair of tokens which have sameCell relationship. E) samceCell=0, Pair of tokens which do not have sameCell relationship.

with respect to a Cartesian plane centered at the top-left corner of the document page. We used an off-the-shelf library to get the content in a PDF document inside a given region, that, along with the coordinates of characters $(x, y)$, provides the font style for every character in the document. The characters along with their coordinates and font styles are further aggregated into tokens, by using the white-space and new-line characters as delimiters.

With the help of table location and the location of individual tokens in the document, only those tokens which are within the given table location are collected by comparing their corresponding coordinate values. Specifically, all the tokens, whose $x$ coordinate is between start $X$ and end $X$ and whose $y$ coordinate is between startY and $e n d Y$ are collected as table text tokens.

After collecting all the tokens from table text using the table coordinates, we generate the training data for the binary relationship classification. Training data requires a pair of tokens and a target label indicating whether or not those two tokens belong to the same cell or not. Once we have a list of all the tokens that are part of the table, for every token, we create a pair of current token with every token, which is located within an imaginary rectangular window around the current token. The size of this imaginary rectangular window will help us determine the number of pairs of tokens to generate.

Training sample is a vector of all the features of a pair of tokens as denoted by 1 .

$$
V=\left[W_{1} F_{i}, W_{2} F_{i}, \text { sameCell }\right]
$$

Where, $W_{1} F_{i}$ are $n$ features of first token, $W_{2} F_{i}$ are $n$ features of second token, and sameCell is the target class indicating True if the two tokens belong to the same cell, False otherwise (see Figure $1)$.

The target class, sameCell is captured using $D a$ toin's WYSIWYG annotation tool, that allows to select a sequence of words on the PDF document and tag those words as a table cell. The training data is generated using the annotated PDF documents and the target label sameCell is assigned accordingly for all the pair of words.

\section{Cell Relationship Features}

For each token in the table, we generate a set of locational and visual features. Use of semantic fea- 
tures of tabular data along with the mentioned features of this work can be one of the future works with the intention of improving the accuracy of the system.

We group the features used in the relation learning task as four categories as below.

Location and Tile features(LTF). The absolute location of a token is important evidence to indicate that it is indeed part of the table. Along with the absolute location, a more generalized positional information of tokens relative to the document makes the contextualization and localization of tokens easier for a reader.

To capture these relative location and distance information, for each token in the table, we consider $(x, y)$ of starting of the token, and $(x, y)$ of the ending of the token in documents (see Figure 1). In order to incorporate contextual information about a token, we split the entire document page into an imaginary matrix of tiles of size $(n X \mathrm{~m})$ and for each tile, we assign a tile number. For each table token, based on its coordinates we find in which tile the token is located, and we include its tile number, the row, and columns of the tile as features of that token (see Figure 1).

Neighborhoodfeatures(NF). The position of the surrounding tokens of a given token indicates the relative position of a token to its neighbors and captures the empty spatial distance around a token. For a given table token, we find a list of $n$ nearest tokens in all the four directions, left, right, top and bottom based on the neighboring tokens' spatial distances with respect to the current token (see Figure 1). The horizontal and vertical relative distances between these neighboring tokens are used as features. The Location and Tile features of neighboring tokens are also included as part of the given token's feature set. This feature ensures that there exists a chain of transitive dependency connecting all the tokens of a cell.

Clustering and Alignment features $(C A F)$. A human reader makes use of the relative closeness and horizontal and vertical alignments of a given word, especially when a table is not completely bordered, to decide which cell the word belongs to. The proximity of a pair of tokens and the presence of neighboring tokens for each token in four different directions captures the information about the relative closeness.

Among all the neighborhood tokens, we identify whether a given token is nearer to the left neighbor or right neighbor. Similarly, we identify whether that token is nearer to the top neighbor or bottom neighbor (see Figure 1). We have used the absence of neighborhood tokens as a set of four categorical features as well, indicating whether or not a given token has left, right, top and bottom neighbor token.

Type and Style features(TSF). Another significant visual clue used by humans in determining whether two words belong to the same cell or not is the content and the styles used in the words. A binary feature representing whether a token is a number or not was used to capture the data similarity within a row or a column. For every pair of tokens, the comparison of font size and bold styles are used to indicate whether the two tokens have a similar font style or not. Use of semantic features of the content of words could be another important clue in differentiating the words into cells.

We find that Neighborhood, Clustering and Alignment features play a critical role in distinguishing the tokens that do not belong to the same cell. All of the feature generation techniques are based on the coordinates of each of the tokens and the coordinates of the table itself. The number of tiles and the number of neighboring tokens are the parameters which can be tuned to achieve better table structure recognition accuracy.

\subsection{Relation Classification Using Multilayer Feedforward Neural Network}

We have used a Multilayer Feedforward Neural Network to model the binary relationship classifier in the experiments. In order to learn the complex patterns that exist in the table layouts, and generalize these patterns we decided that deep learning is the right tool. Working at token-level, we have huge training data as well and deep neural networks seemed a right candidate for the task.

The generated training data is fed into the Multilayer Feedforward Neural Network that uses relu activation function in the hidden layers and a sigmoid activation function in the output layer. The models were trained using Adam optimizer and $B i$ nary cross-entropy loss function as defined in 2 (Zhang, 2019).

$$
\text { Loss }=-[y \log (p)+(1-y) \log (1-p)]
$$

Where $y$ is a binary indicator of correct prediction of a sample, $p$ is the predicted probability for a training sample. 


\begin{tabular}{|l|l|}
\hline Feature Parameter & Value \\
\hline Number of left, right, top and bottom neighbor tokens & 1 \\
Window size for token pair generation & $30 \times 30$ pixels \\
Number of tiles & 20 tile rows x 20 tile columns \\
\hline
\end{tabular}

Table 1: Word-level feature generation parameters

The input feature vector of $N$ dimension is fed into the network and the sigmoid output value is decoded as binary classes, 0 indicating that the two tokens do not belong to the same cell, 1 indicating that the two tokens belong to the same cell. In our experiments, the Multilayer Feedforward neural network has been built using Keras backed by Tensorflow, for quick experimentation and development.

\section{Experiments and Results}

\subsection{Dataset and Evaluation Metrics}

Due to the lack of publicly available datasets that suit our methodology, we prepared the training data on our own. The dataset used for the experiments contains a total of 250 PDF documents, having one table per document. We ensured that the tables present in our dataset represent the possible diverse type of tables that are most commonly used. Our dataset has tables with and without borders, with and without column headings, with column and row spans, with all types of text alignments, varying line, and word spacing, and font styles. All the PDF documents were annotated using Datoin's WYSIWYG annotation tool.

Using the parameters listed in Table 1, we created approximately 0.3 million training samples from all the tokens of 250 tables, containing 83 different features. Training samples are split by a 9 to 1 ratio for training and testing, keeping approximately 30,000 samples for testing.

\begin{tabular}{|l|l|l|}
\hline & Training data & Test Data \\
\hline True Class & 60,000 & 9,000 \\
False Class & $2,10,000$ & 21,000 \\
\hline Samples size & $2,70,000$ & 30,000 \\
\hline
\end{tabular}

Table 2: Approximate distribution of target labels

The distribution of target labels in our training and testing dataset is shown in Table 2. The imbalance in the distribution of classes makes sense because for every token in the table, within an imaginary rectangular window around that token, the number of tokens that are in the same cell will be less than the number of tokens that are not in the same cell.

Measuring how many predicted cells are actual cells in a given table, would be a more explanatory metric for evaluation. However, if one token among all the tokens of a cell is wrongly predicted by the relationship classification model as belonging to a different cell, then measuring the correctness of this prediction at a cell-level would be challenging. So we decided to use the accuracy of the binary classification model itself as our evaluation metric. This token-level metric is simpler and straightforward.

\subsection{Hyperparameters}

We have experimented with the hyperparameters of the neural network architecture itself. Table 3 defines the set of hyperparameters used in our experiments. In terms of the number of weights, Set$l$ is a simpler network with fewer weights to learn and Set-2 is a more complex network.

\begin{tabular}{|l|l|l|}
\hline Hyperparameter & Set-1 & Set-2 \\
\hline Number of layers & 4 & 5 \\
Number of Epochs & 200 & 300 \\
Batch size & 300 & 100 \\
Learning Rate & 0.001 & 0.001 \\
\hline
\end{tabular}

Table 3: Hyperparameters used in Multilayer Feedforward Neural Network

\begin{tabular}{|l|l|}
\hline Feature Set & Features \\
\hline LTF & Location and Tile features \\
\hline NF_CAF & $\begin{array}{l}\text { Neighborhood features, } \\
\text { Clustering and } \\
\text { Alignment features }\end{array}$ \\
\hline TSF & Type and Style features \\
\hline
\end{tabular}

Table 4: Feature sets used in the experiments

It is important to note here that a smaller batch size and a higher number of epochs do increase the F1-Scores and help the model to learn more com- 


\begin{tabular}{|lllll|}
\hline Feature Set & Class & Precision & Recall & F1-Score \\
\hline LTF & True & $77.89 \%$ & $91.33 \%$ & $83.51 \%$ \\
& False & $95.13 \%$ & $85.91 \%$ & $90.29 \%$ \\
\hline LTF \& NF_CAF & True & $93.65 \%$ & $89.50 \%$ & $91.07 \%$ \\
& False & $94.07 \%$ & $96.10 \%$ & $95.73 \%$ \\
\hline LTF, NF_CAF \& TSF & True & $\mathbf{9 4 . 1 0 \%}$ & $\mathbf{9 0 . 8 5 \%}$ & $\mathbf{9 2 . 6 2 \%}$ \\
& False & $\mathbf{9 7 . 5 6 \%}$ & $\mathbf{9 8 . 2 7 \%}$ & $\mathbf{9 8 . 1 5 \%}$ \\
\hline
\end{tabular}

Table 5: Results of binary relation classification using Hyperparameter Set-2

plex patterns in the data, at the cost of increased training time.

\subsection{Experiments}

We have experimented with many combinations of feature sets and feature generation parameters and selected the best three sets of features, as listed in Table 4. The Neighborhood features and Clustering and Alignment features are combined into one set because both the features measure the togetherness of two given table tokens.

In each of these experiments, we have further experimented with 2 sets of neural network hyperparameters listed in Table 3.

\subsection{Results and Discussion}

All the experiments with the two sets of neural network hyperparameters listed in Table 3, indicated that Set-2 outperforms the Set-1. So, we have listed only the results of experiments carried out using hyperparameters Set-2 in Table 5.

Multiple experiments indicated that neighborhood features have sufficient information to capture the table structure and the use of visual clues does increase accuracy. However, one experiment showed that increasing the number of neighboring tokens for each token, reduces the Recall measure of True class. Increased number of False classes could be a possible explanation for this behavior. Also, increasing the number of hidden layers or hidden units of the network did not improve the accuracy further.

The model achieved an overall accuracy of $97.95 \%$ on the test set after training the network for about an hour. Clearly, the model is predicting the cell-relationships on unseen token pairs with very high accuracy. A set of 20 documents containing a variety of tables, which are not part of training documents, are considered as a validation set.

The F1-Score of False class is much better than that of True class. One possible reason for this could be, the tokens that are not likely to be in the same cell will clearly have a distinguishable set of locational and neighboring features. It is clear that the recall of True class is causing the F1-Score to be low. Our training dataset has comparably fewer training samples for the True class this could be a possible reason for the low recall scores.

Manual verification of individual table cells with the prediction of the relation classifier shows that the model is able to generalize the cell recognition task across a variety of table cells. The cell relationships are identified accurately irrespective of the presence of borders lines, column, and row spans and text alignments. The relationship among the tokens of a table is learned by the model based on Neighborhood, Clustering and Alignment features of the tokens. However, for a few tokens where the neighborhood features do not have a clear separation with tokens from nearby cells, the model combines the tokens from adjacent cells, producing wrong predictions. Because of the absence of visual separation among the tokens of two closely aligned cells, the model predicts those multiple cells as a single cell.

\section{Conclusion and Future Work}

By applying the idea of relation extraction in table structure recognition task, we have shown the possibility of high accuracy information extraction in unstructured documents. Table structure recognition as relation extraction task is a novel approach in table extraction process and to the best of our knowledge has never been explored. We have taken the first step towards this direction and have proved that a bottom-up approach of cell relationship extraction is the right way towards table structure recognition task. We have compared three sets of features and showcased the significance of cognitive features in our experiments.

For a few of the tables, closely aligned adja- 
cent cells are wrongly identified as one cell. Incorporating the semantic features of the content of the words, especially using Natural Language Processing, will enrich the feature vector and should help the model to do better generalizations. Exploring the different layout and visual features and improving the accuracy of the proposed method could be one of the possible future works.

Building on top of cell-relationship recognition work, we hope to explore the table structure extraction further. The knowledge of table cells can be used to build up the rest of the table structures from bottom-up. We believe that the relation extraction methodologies apply to other document understanding tasks and we hope to explore them as well.

\section{References}

Rahul Anand, Hye-Young Paik, and Cheng Wang. 2019. Integrating and querying similar tables from pdf documents using deep learning. CoRR abs/1901.04672.

S. Clinchant, H. Djean, J. Meunier, E. M. Lang, and F. Kleber. 2018. Comparing machine learning approaches for table recognition in historical register books. In 2018 13th IAPR International Workshop on Document Analysis Systems (DAS). pages 133138. https://doi.org/10.1109/DAS.2018.44.

Andreiwid Correa and Pr-ola Zander. 2017. Unleashing tabular content to open data: A survey on pdf table extraction methods and tools. pages 54-63. https://doi.org/10.1145/3085228.3085278.

Azka Gilani, Shah Rukh Qasim, Imran Malik, and Faisal Shafait. 2017. Table detection using deep learning. https://doi.org/10.1109/ICDAR.2017.131.

M. Gbel, T. Hassan, E. Oro, and G. Orsi. 2013. Icdar 2013 table competition. In 2013 12th International Conference on Document Analysis and Recognition. pages 1449-1453. https://doi.org/10.1109/ICDAR.2013.292.

L. Hao, L. Gao, X. Yi, and Z. Tang. 2016. A table detection method for pdf documents based on convolutional neural networks. In 2016 12th IAPR Workshop on Document Analysis Systems (DAS). pages 287-292. https://doi.org/10.1109/DAS.2016.23.

Isaak Kavasidis, Sergio Palazzo, Concetto Spampinato, Carmelo Pino, Daniela Giordano, Daniele Giuffrida, and Paolo Messina. 2018. A saliency-based convolutional neural network for table and chart detection in digitized documents. CoRR abs/1804.06236.

Stefan Klampfl, Kris Jack, and Roman Kern. 2014. A comparison of two unsupervised table recognition methods from digital scientific articles. D-Lib Magazine https://doi.org/10.1045/november14-klampfl.

Bernhard Krüpl and Marcus Herzog. 2006. Visually guided bottom-up table detection and segmentation in web documents. In $W W W$.

Shantanu Kumar. 2017. A survey of deep learning methods for relation extraction. CoRR $\mathrm{abs} / 1705.03645$.

Martha O. Perez-Arriaga, Trilce Estrada, and Soraya Abad-Mota. 2016. Tao: System for table detection and extraction from pdf documents. In FLAIRS Conference.

S. Schreiber, S. Agne, I. Wolf, A. Dengel, and S. Ahmed. 2017. Deepdesrt: Deep learning for detection and structure recognition of tables in document images. In 2017 14th IAPR International Conference on Document Analysis and Recognition (ICDAR). volume 01, pages 1162 1167. https://doi.org/10.1109/ICDAR.2017.192.

Alexey Shigarov. 2015. Table understanding using a rule engine. Expert Systems with Applications 42:929937. https://doi.org/10.1016/j.eswa.2014.08.045.

Alexey Shigarov, Andrey Mikhailov, and Andrey Altaev. 2016. Configurable table structure recognition in untagged pdf documents. https://doi.org/10.1145/2960811.2967152.

Burcu Yildiz, Katharina Kaiser, and Silvia Miksch. 2005. pdf2table: A method to extract table information from pdf files. pages 1773-1785.

Jiawei Zhang. 2019. Gradient descent based optimization algorithms for deep learning models training. CoRR abs/1903.03614. 